\title{
Erratum to: Modeling the effects of biomass accumulation on the performance of a biotrickling filter packed with PUF support for the alkaline biotreatment of dimethyl disulfide vapors in air
}

\author{
Luis Arellano-García • Antonio D. Dorado • \\ Axayacatl Morales-Guadarrama $\cdot$ Emilio Sacristan • \\ Xavier Gamisans • Sergio Revah
}

Published online: 1 February 2015

(C) Springer-Verlag Berlin Heidelberg 2015

\section{Erratum to: Appl Microbiol Biotechnol DOI 10.1007/s00253-014-5929-7}

The article entitled "Modeling the effects of biomass accumulation on the performance of a biotrickling filter packed with PUF support for the alkaline biotreatment of dimethyl disulfide vapors in air".

Appl Microbiol Biotechnol (2015) 99:97-107. DOI 10.1007/s00253-014-5929-7.

The online version of the original article can be found at http://dx.doi.org/ 10.1007/s00253-014-5929-7.

\section{Arellano-García}

Departamento de Ingeniería de Procesos e Hidráulica, UAM,

Iztapalapa, San Rafael Atlixco 186, Col. Vicentina, Iztapalapa,

Mexico City 09340, Mexico

\section{A. D. Dorado $•$ X. Gamisans}

Departamento de Ingeniería Minera y Recursos Naturales, UPC, Av.

Bases de Manresa 61-73, 08242 Manresa, Barcelona, Spain

A. Morales-Guadarrama $\cdot$ E. Sacristan

Departamento de Ingeniería Eléctrica, UAM Iztapalapa, San Rafael

Atlixco 186, Col. Vicentina, Iztapalapa, Mexico City 09340, Mexico

\section{A. Morales-Guadarrama $\cdot$ E. Sacristan}

Centro Nacional de Investigación en Imagenología e Instrumentación Médica, UAM Iztapalapa, San Rafael Atlixco 186, Col. Vicentina, Iztapalapa, Mexico City 09340, Mexico

\section{S. Revah $(\bowtie)$}

Departamento de Procesos y Tecnología, UAM Cuajimalpa,

Artificios 40, Col. Hidalgo, Álvaro Obregón, Mexico City 01120,

Mexico

e-mail:srevah@xanum.uam.mx
In the mathematical model section, page 100 , equation number 7 , should be

$\frac{\partial \mathrm{C}_{\mathrm{B}}}{\partial \mathrm{t}}=\mathrm{D}_{\mathrm{eff}} \frac{\partial^{2} \mathrm{C}_{\mathrm{B}}}{\partial \mathrm{x}^{2}}-\mathrm{R}_{\mathrm{B}}$

Published version of the article contains a typographical error in equation 7 where the term $\delta^{-2}$ was included by mistake. In any case this error was not present in the mathematical simulation. 